\title{
An eLearning Standard Approach for Supporting PBL in Computer Engineering
}

\author{
Rocio Garcia-Robles, Fernando Diaz-del-Rio, Saturnino Vicente-Diaz, and Alejandro Linares-Barranco
}

\begin{abstract}
Problem-based learning (PBL) has proved to be a highly successful pedagogical model in many fields, although it is not that common in computer engineering. PBL goes beyond the typical teaching methodology by promoting student interaction. This paper presents a PBL trial applied to a course in a computer engineering degree at the University of Seville, Spain. To promote the reusability and interoperability of the PBL trial, a design-driven approach was used, based on the Internet protocol Multimedia Subsystems (IMS) Learning Design (LD) standard. This paper presents the outcomes of using this method to innovate teaching practices in a blended learning environment. Design and implementation results, as well as users' opinions, are presented and analyzed.
\end{abstract}

Index Terms-Blended learning, e-learning standards, educational methodology, educational technology, engineering education, IMS-LD, learning design (LD), learning systems, learning management system (LMS), Moodle, problem-based learning (PBL).

\section{INTRODUCTION}

$\mathbf{P}$ ROBLEM-based learning (PBL) is the contextual, collaborative, and constructivist learning environment "par excellence" [1], and has long been used in higher education (HE) institutions, especially in medical training. However, it is harder to find documented PBL trials in computer engineering-related backgrounds. The current paper describes such a trial, based on eLearning standards, being undertaken by the authors in the Higher Technical School of Computer Engineering (HTSCE) at the University of Seville (US), Spain.

Although applications of PBL vary, it has three essential characteristics: problems as a stimulus for learning, tutors as facilitators, and group work as stimulus for interaction [2]. Pro-PBL practitioners argue that this model has the potential to prepare students more effectively for future learning, because it is based on four modern insights into learning: constructive, self-directed, collaborative, and contextual [2]. However, the model's detractors concluded that PBL graduates showed potentially significant gaps in their cognitive knowledge base and did not demonstrate expert reasoning patterns, and that PBL was very costly [3]. Others took a middle position and

This work was supported in part by the Spanish Science And Education Ministry Research Project TIN2006-15617-C03-03 and by the Andalusian Government Excellence Research Project P06-TIC-02298.

The authors are with the Department of Computer Architecture and Technology, University of Seville, Seville, Spain (e-mail: satur@atc.us.es). concluded that PBL had a robust positive effect on skills but a negative effect on knowledge [4].

According to experts in this field, design-based research is needed which blends empirical educational research with the theory-driven design of learning environments to help educators understand how, when and why educational innovations work in practice [5]. A promising approach is the model-based one supported by IMS-Learning Design (IMS-LD) [6], which is a conceptual model (ontology) for the description of teachinglearning processes [7]. In this way, the PBL approach is made explicit, subjecting it to review and change.

Information and Communications Technologies (ICTs) can be very useful for supporting PBL trials by providing a richer learning environment, offering students alternative ways of gaining knowledge and information, enabling more accurate assessment, and individualizing practice, feedback and reflection [4]. Current e-learning systems, however, offer limited support for an implementation of PBL combined with the use of e-learning standards. Nevertheless the use of such standards may prove to be a very useful methodology in terms of conceptualization and design of the pedagogic trial, even though further improvements are necessary for its implementation and exploitation.

This paper presents a PBL trial in a computer engineering course. The trial's design is based on the IMS-LD standard, and is implemented using Moodle [8], a popular web-based course or learning management system (LMS). The main aims were to support an innovative pedagogic approach in a blended learning environment (face-to-face learning plus eLearning), as well as adopting standard model-based methodologies in a prospective approach designed to make the PBL trial easily assessable and reusable. Users' opinions (both students and teachers) were also analyzed with two questionnaires. Finally, the main conclusions, and the next stage for this work, are presented.

\section{Context: The Relevance of The Adoption of PBL}

The current syllabus for Computer Engineering of the HTSCE at the University of Seville was adopted fairly recently. Following a transition period during the courses held in the academic years 1997-1998 and 1998-1999, the 1999-2000 course was the first year when the new syllabus was followed completely. The course is divided into two four-month terms, which produces a structural problem of "atomization:" the organization of the syllabus requires students to study a lot of small course elements, which are not related to each other in a way that controls the learning flow. Apart from this problem of organization, there are several academic issues that need 
addressing, such as coordination between courses taught, adequate subject knowledge on the part of teachers, coordination between departments and lecturers in charge of courses, overlap between courses, and so on. Further problems arise from factors such as the excessive number of tests or exams that students have to take in one four-month term, forcing them to skip classes to study for those exams, or the coordination problems caused by the holidays.

Prerequisites are generally not required for a student to take a course. Consequently, many students' choice of course depends more on how easy the course is, and/or timetable considerations, rather than content or organization. As a result students can accumulate an unrelated collection of courses, which end up like isolated watertight compartments. The loser here is clearly specialization.

The Declaration of Bologna [9] has started a process to promote the convergence between the national educational systems of European Union member countries, to support the development of a European Higher Education Area (EHEA) by 2010. The goal of creating this educational space is to establish easily comparable systems of degrees, to facilitate academic and professional recognition in the EU. The higher education systems of the different countries must be harmonized and they must adapt the structure, content, and definition of skills on which learning results are based.

Current Spanish education legislation divides degree syllabuses into credits. A credit is defined as the unit of accumulation that measures the hours of classes (theory classes and laboratory classes, but never study time). One credit is equal to 10 hours. An important point to take into account is the difference between theoretical and laboratory classes in the amount of work involved for students.

Bologna is promoting the change from knowledge-based syllabuses to competence-oriented ones; it therefore seems appropriate to use constructivist-learning methodologies. Technical degrees are faced with particular problems in adapting teaching to this philosophy. First, little experience has been gained with constructivist methods, because many of these degrees have only recently been implemented. Second, the teachers are currently immersed in the transition period, so traditional and new teaching approaches have to coexist during the transition period. This reality implies a high cost in terms of both material and human resources. These difficulties are aggravated by the high student-teacher ratio, particularly in teaching engineering degrees.

The study described in this paper was carried out within an engineering degree. The department in charge of this course is also responsible for other technical engineering courses in the area of electrics and electronics, such as Industrial Robotics and Industrial Informatics, where this approach is also being adopted. This paper presents the results of applying this method in a course "Parallel System Architecture 1" (ASP1). This fourth-year course studies advanced electronics for computer architecture for modeling and simulating complex and parallel architectures of computers and computer networks. This course is worth 4.5 classical credits ( 3 theoretical and 1.5 laboratory) and is taught in four groups of 90 students each for theoretical classes, and 15 groups of 24 students each for laboratory classes. Three lecturers are in charge of teaching both theoretical and lab classes. Clearly the high student/lecturer ratios, and a shortage of human resources, make it almost impossible to apply this kind of technique across the whole course, both theory and labs. This problem is exacerbated by teachers having to divide their time between teaching and research activities, as in many other universities.

A final consideration is that the use of these methods should not result in cognitive gaps in the students who learn with this methodology, as stated in Section I [3]. This factor is very important in this trial, because ASP1 must convey the basic knowledge in the field if students are to understand the following course (ASP2).

These considerations raise the question: how can the new teaching model/technique suitably be integrated (using new pedagogical approaches) into the current syllabus? Therefore, it was decided to apply this new trial to a reduced number of groups of students for laboratory classes only. The remaining students attended the classical lab classes, and all students attended the theoretical lectures.

\section{Design of the PBL BASEd on E-LEARning Standards}

\section{A. Introduction to Learning Design (LD)}

The LD specification is based on the Educational Modeling Language, originally developed at the Open University of the Netherlands (OUNL).

The specification aims to represent the "learning design" of "units of learning" (UoL), in a semantic, formal and machine-interpretable way [10]. A UoL can be any instructional or learning event of any granularity, for example a course, a workshop, a lesson or an informal learning event. A "learning design" is defined as the description of the teaching-learning process that takes place in the UoL. The key principle in LD is that it represents the learning activities and the support activities that are performed by different persons (learners, teachers) in the context of a UoL. These activities can refer to different learning objects that are used during the performance of the activities (e.g., books, articles, software applications, pictures), and it can refer to services (e.g., forums, chats, wiki's) that are used to collaborate and to communicate in the teaching-learning process. [11]

The LD specification can be seen from (at least) four different perspectives: 1) an educational modeling language; 2) an eLearning methodology; 3) a set of applications; and 4) an interoperability specification [12].

The teachers involved in this PBL trial have concluded that the IMS-LD specification is a very useful methodological tool for formalizing the design of the PBL pedagogical scenario. In fact, they have used the methodology included in the IMS Learning Design Best Practice and Implementation Guide (LDBPIG) [13], as explained here.

The aforementioned specification was developed to meet some specific requirements: Completeness, Pedagogical expressiveness, Personalization, and Compatibility [7]. Completeness means that the specification must be able to describe fully the teaching-learning process in a UoL. Pedagogical expressiveness implies that, while the specification must be sufficiently flexible to describe LDs based on all kinds of 
pedagogies, it must avoid biasing designs towards any specific pedagogical approach [7].

The IMS-LD specification enables flexible and sophisticated pedagogical approaches by providing support for: 1) multiple, as well as single, learners and their coordination; 2) a wide range of present, as well as future, pedagogical models; 3) learning activities and learning services, as well as content [12].

In the framework of the department of the University of Seville in which the PBL trial has taken place, completeness and pedagogical expressiveness are considered key issues for several reasons. First, because blended learning solutions are required, and IMS-LD can support them. Second, because the result of this trial will probably be applied to other courses, whose educational approach may differ, pedagogical flexibility and neutrality are required. Third, the teachers involved in this trial are interested in supporting social constructivist approaches such as PBL, and IMS-LD is suitable because it supports multiple learner collaboration.

\section{B. PBL Design Using LD}

The LD specification consists of several components, one of them being the IMS-BPIG [13]. According to those guidelines, the design and development of education is an incremental process that systematically follows the stages of analysis, design, development, implementation, and evaluation. The following steps must be taken in order to proceed from a description of an educational problem to a learning scenario.

1) The analysis phase should result in a didactical scenario that is captured in the form of a narrative.

2) The narrative of the analysis stage is taken and cast in the form of a series of (nested) UML activity diagrams. The UML diagrams capture the workflow aspects of the narrative.

3) An XML document instance for the UoL is formulated on the basis of the UML activity diagrams. Any XML document instance should be valid against the LD Specification.

Using LD solely as a methodology for supporting the design of the PBL scenario involved undertaking steps 1 and 2. The corresponding roles' description, narrative and UML activity diagrams are presented here.

The PBL scenario features three Case Studies (CS), each of which consists of three milestones. The different roles involved in each CS are as follows.

Teachers' roles:

- Facilitator (FA): In principle, all the teachers (three in these three CS) play the role of Facilitator. When adopting a PBL pedagogical approach, the role of the teacher is mainly that of stimulating students in their constructivistic experience. So, the terms "teacher" and "facilitator" are considered to be equivalent.

- Coordinator $(\mathrm{CO})$ : One of the facilitators will play the Coordinator role in each of the CS. The coordinator has to design the CS description and assess students' and teachers' opinions of both the PBL methodology and the CS implementation.

- Evaluator (EV): At least one of the facilitators will play the Evaluator role in each of the CS. The evaluator is responsible for assessing and evaluating group and individual proficiency. Nevertheless, Facilitator and Coordinator are also partially involved in the evaluation. The aforementioned teachers' roles can be played by the same person or by several different persons. In this PBL trial, the Coordinator and Evaluator were played by the same teacher in each CS, and the Facilitator role was played by two teachers in each CS. An additional teacher undertook just one of the coordinator's tasks, as an external observer evaluating learners' and teachers' opinions about PBL methodology and CS implementation.

Students' roles:

— Student (ST): In principle, all the students play such a role. But, in order to promote organizational skills, the students are asked to play different roles (see later) in each CS. This role implementation also benefits the FAs, because it scales the communication workload between students and the Facilitator.

- Chairperson (CP): On behalf of the whole group, s/he is in charge of the communication with external partners (other groups' Chairpersons and the Facilitator). The chairperson, the spokesperson for the group, is responsible for recording key group decisions, and the chosen representative must be appointed as such by the facilitator.

- Speaker (SK): S/he is the person who must present the outcomes of the final CS solution in the corresponding final face-to-face presentation session. S/he must also discuss with the rest of the Chairpersons and Speakers at this session.

The three students from each group rotate roles in each of the three CS, ensuring that no student is burdened with the resolution of the whole CS.

The following narrative and UML diagrams are used to formalize the design of the PBL trial, with annotations describing the corresponding environments used in the context of the LMS. Fig. 1 represents the global diagram and Figs. 2-8 provide the detailed elements.

As shown in the global UML diagram (see Fig. 1), the PBL scenario consists of two initial activities ("Preparatory work," "Logistical tasks"), and a composite activity for each CS. This last is a compound of a composite activity for each CS milestone, and three more activities ("Global CS delivery," "Global CS face-to-face session," "Global CS evaluation"). The CS milestone composite activity consists of two activities ("Students' group work" and "CS milestone face-to-face session"). Both CS and CS milestone composite activities are iterative (indicated by the $*$ in the UML activity diagram).

A detailed description of the activities workflow follows:

1. Preparatory work: Facilitators choose coordinators and evaluators among themselves; the Coordinator elaborates the CS description and presents it to the Facilitator and Evaluator; they read the CS description and comment on it; the Coordinator improves the CS description and makes it available again to the Facilitator and Evaluator. This process is repeated as many times as necessary until 


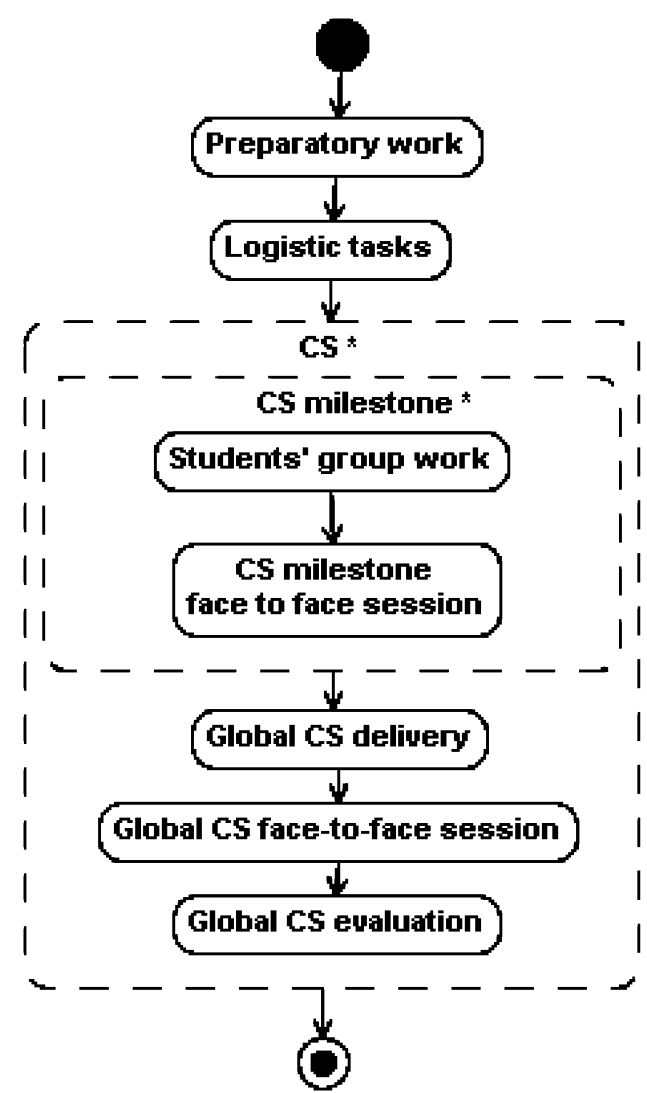

Fig. 1. Global PBL.

the CS description is agreed upon among the Facilitator, Evaluator and Coordinator (see Fig. 2).

2. Logistical tasks: First, the Facilitator makes the PBL methodology description available to students, and selects students to be involved in the PBL trial, based on a questionnaire to be answered by the students in a face-to-face session. This questionnaire covers the PBL methodology and details of the planned CS scheduling. The Facilitator gives the CS_ms1 description to students (by uploading a file to the LMS). Students organize themselves into groups of three, and choose the chairperson for the current CS. The Facilitator provides assistance and appoints groups and the corresponding Chairpersons, (students register themselves in the LMS, and send an e-mail to the Facilitator with the names of the three people from each group; the Facilitator uses the LMS to create the corresponding working groups). One private and one public forum are activated to support communication and discussion at intra- and intergroup levels (see Fig. 3).

3. Problem description delivery: The Coordinator makes the problem description and the first milestone available to the students (by uploading a file to the LMS). In the UML diagram this step is called CS_ms1, which stands for Case Study milestone 1 (see Fig. 3).

4. For every Case Study (see Fig. 4):

4.1 Students work in groups: Every student in the group reads the problem (by downloading the corresponding PDF documents from the LMS). The group then meets (they may the use synchronous or asynchronous facilities of the $L M S$ ) to discuss how to clarify the problem and plan work scheduling. They eventually arrive at their own succinct statement of the problem at hand. Once this is done, the Chairperson states the problem, and interacts with the Facilitator to clarify open issues and agree on a work schedule. Students can use any communication tool available in the LMS, (e.g., chat, e-mail,...) It is important to note that, in order to scale teacher-student interaction, students only communicate with the Facilitator via the Chairpersons, and, the Facilitator creates a frequently asked questions (FAQ) list published in the LMS containing the most frequently encountered and interesting issues arising from the interaction with Chairpersons.

4.2 The Chairperson states the discussed solution (CS_ms1) and uploads it to the LMS before the deadline.

4.3 All the Chairpersons discuss the different submitted solutions (via the LMS). In the meantime, the Facilitator reads the solutions and monitors the Chairpersons' discussion (by using synchronous tools such as the public forum, and asynchronous tools such as e-mail).

4.4 CS_ms1 face-to-face session: The Chairpersons discuss other groups' solutions. Helped by the rest of the students from his/her group, the Chairperson can defend or even rectify their solution. Chairpersons can also ask the Facilitator about issues that have arisen. At the end of the discussion, the Facilitator provides assistance, clarifies, gives some support for further work and finally gives some feedback on group and individual progress. If appropriate (see Fig. 5), the Facilitator gives the solution of the just-finished milestone (by uploading a file to the LMS corresponding to the CS_msl).

4.5 Same as step 3 but for the next milestone (CS_ms2).

4.6 Steps 4.1 to 4.4 are repeated for CS_ms2.

4.7 Same as step 3 but for the next milestone (CS_ms3).

4.8 Steps 4.1 and 4.2 are repeated for CS_ms3.

4.9 The Chairperson summarizes the findings for the whole CS in a few slides. Before the deadline (see Fig. 6), s/he uploads two deliverables using the LMS (CS_ms3 solution and the slides for presenting the CS solution).

4.10 All the Chairpersons discuss the different submitted solutions (by using the LMS). In the meantime, the Evaluator reads the solutions and monitors the Chairpersons' discussion (public forum).

4.11 Overall CS face-to-face session: The Evaluator draws lots to give the speaker role to one of the two students from each group who have not been Chairperson for the current CS. The Evaluator also draws lots to decide order of the presentations. Each Speaker presents the final solution of the current CS. Chairpersons and Speakers discuss other groups' solutions, helped by 


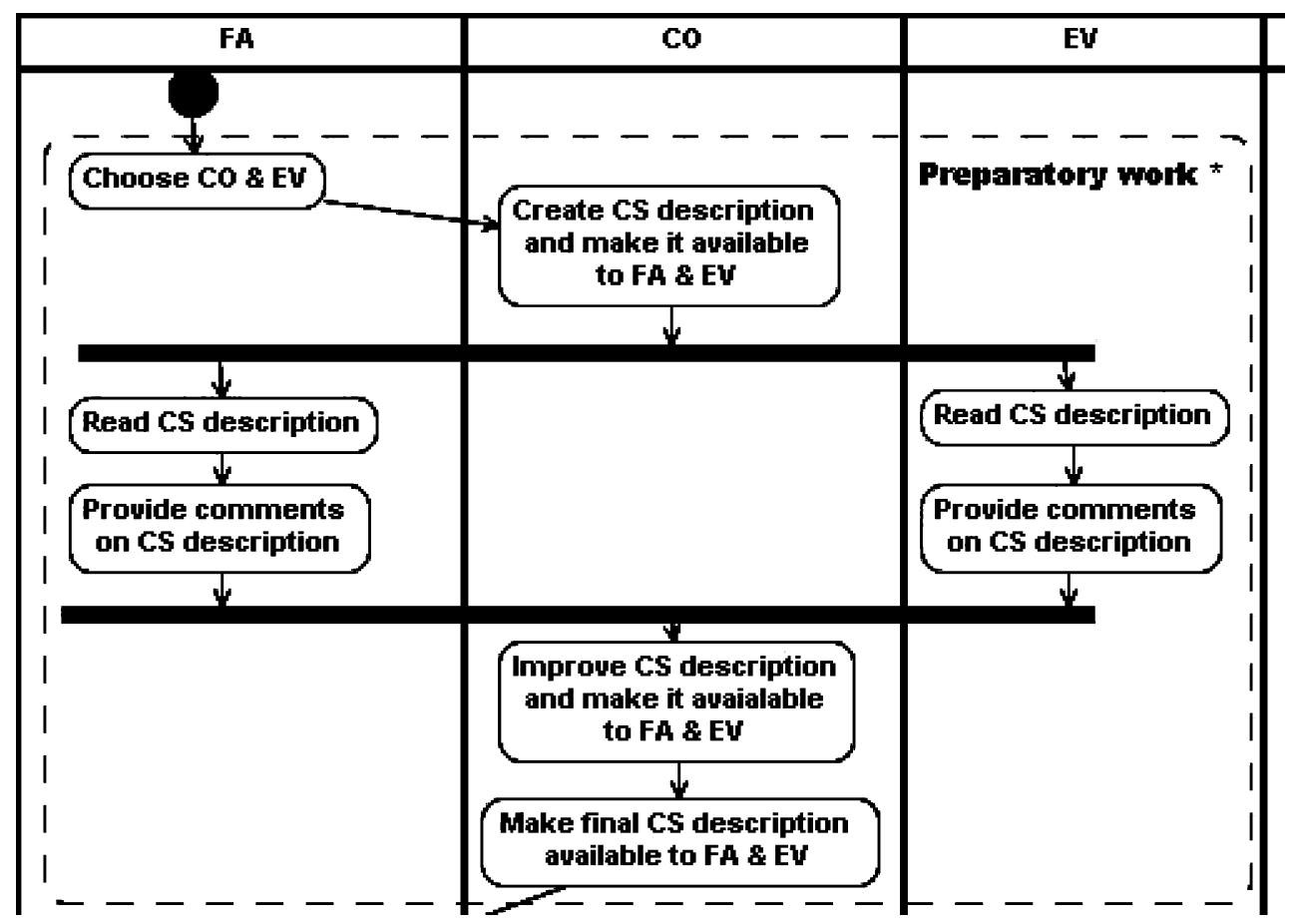

Fig. 2. Preparatory work.

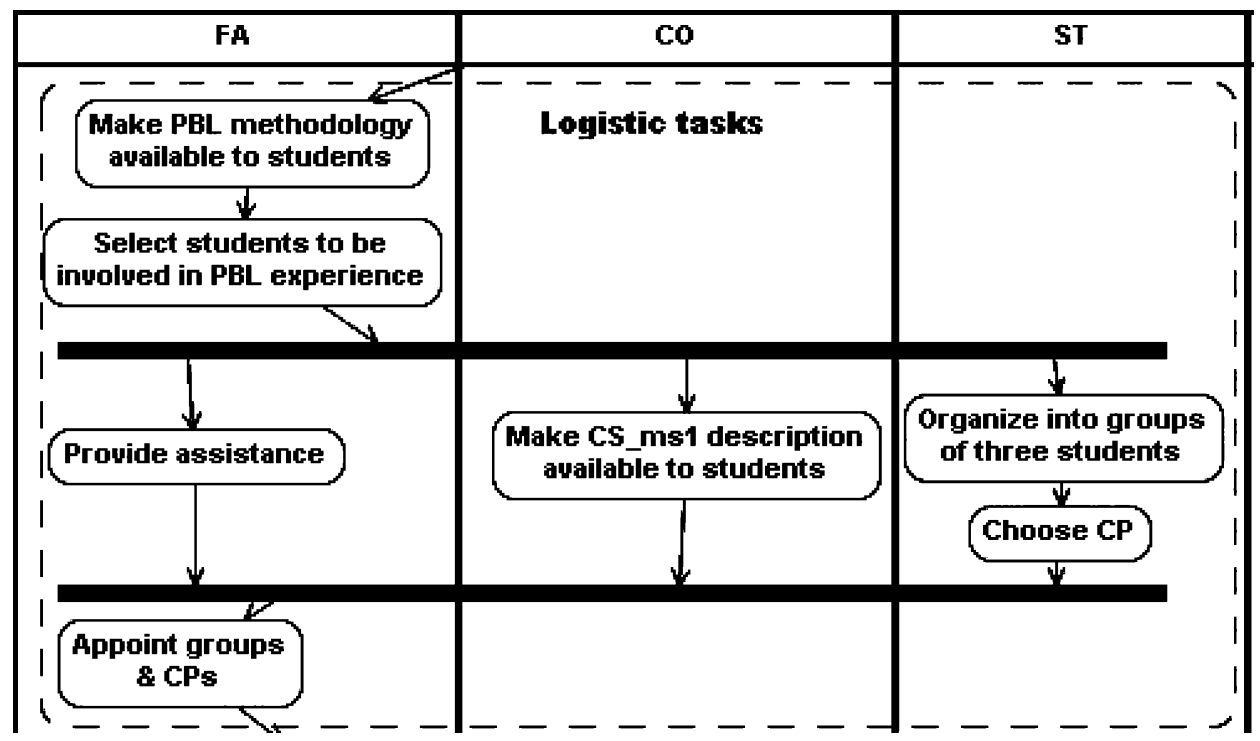

Fig. 3. Logistical tasks.

the other students from their group. At the end of the discussion, the Evaluator provides assistance, clarifies open issues, and finally gives some feedback on group and individual progress (see Fig. 7).

4.12 Evaluation: The Evaluator evaluates the work of the groups, Chairpersons and Speakers. The Coordinator collects students' and teachers' feedback about the PBL trial and how the CS implementation is going (by using a questionnaire available in the LMS). This evaluation is used later to improve the implementation of the next CS. Subsequently, the Facilitator, Coordinator, and Evaluator discuss group performance and individual marks in a face-to-face meeting.
The Evaluator gives marks and justification to groups (in a file uploaded to the LMS); the Evaluator attends students' comments and complaints (by using e-mail or face-to-face meeting); the Evaluator gives students (see Fig. 8) their final marks (in a file uploaded to the $L M S$ ).

\section{Benefits and Drawbacks of Using IMS-LD to Support PBL} Design

There are some important benefits from using IMS-LD for designing a pedagogical scenario. The main ones, in the framework of the PBL trial, are listed here.

LD suggests using a roles model for promoting the distinction between possible roles and real actors. If no formal method- 


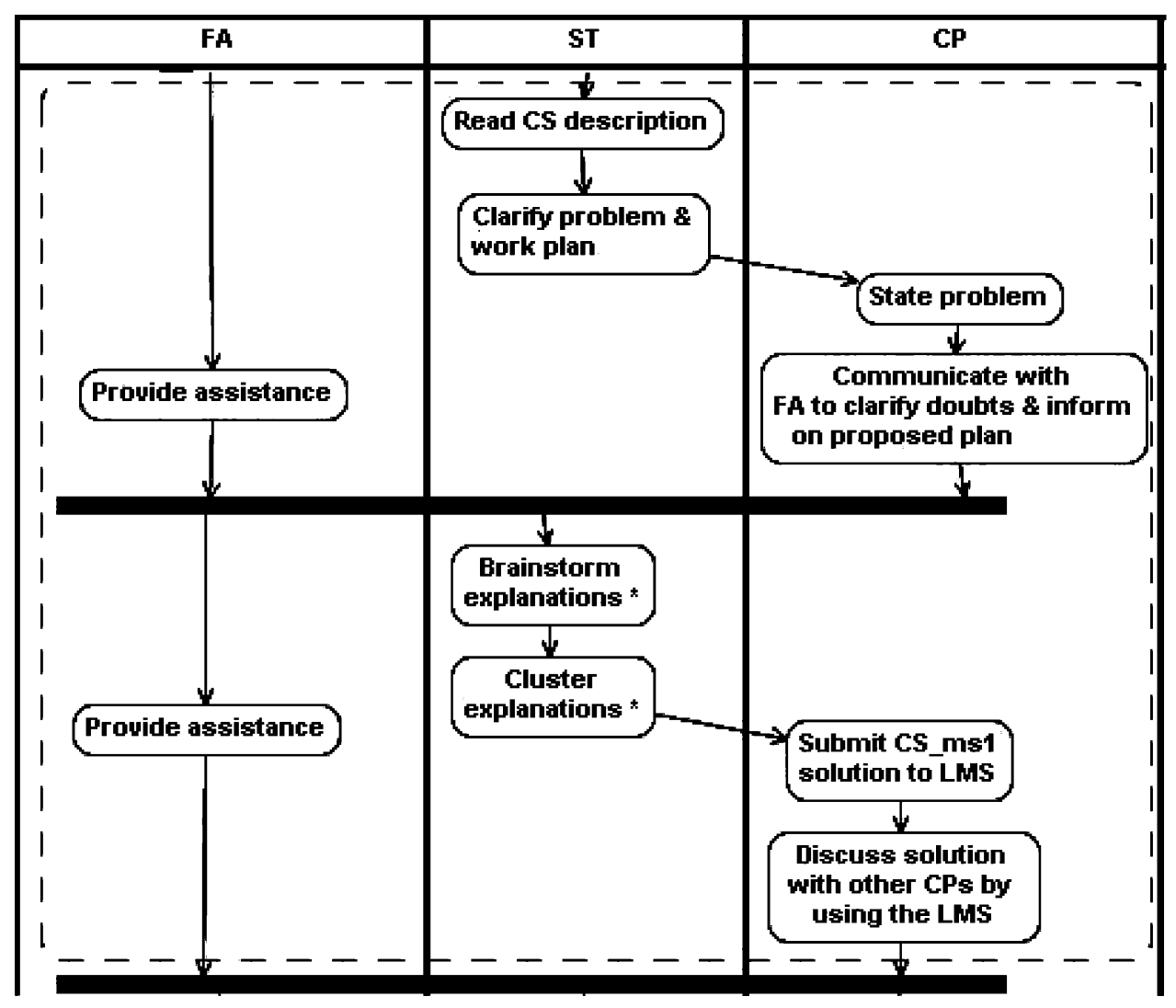

Fig. 4. Students' work in their group.

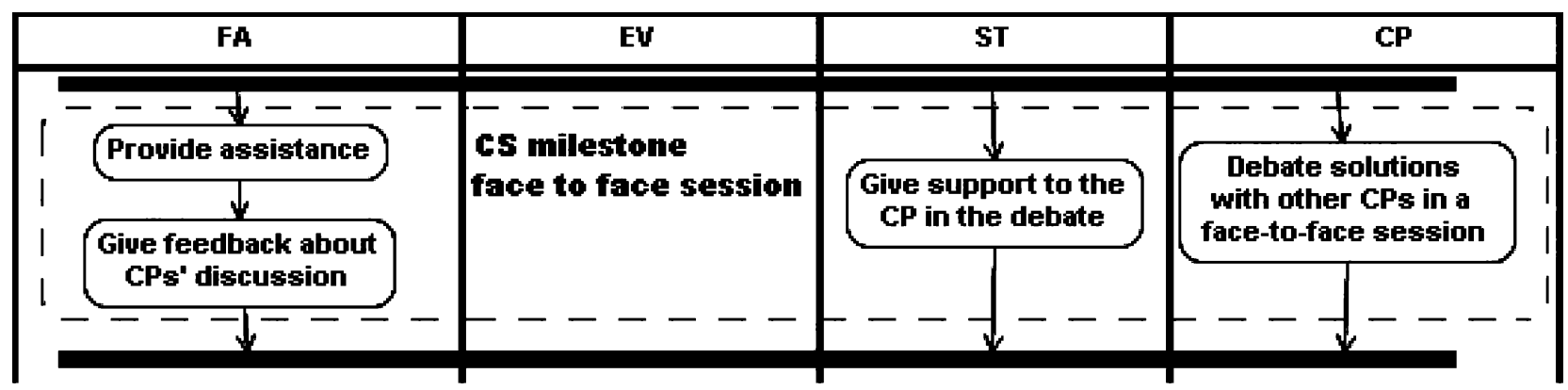

Fig. 5. CS milestone face-to-face session.

ology is followed, there is a risk of considering only the actual actors involved in the learning trial. For example, if there are only two teachers involved in the PBL, responsibilities are split between them. If the number of teachers is subsequently increased, obviously the responsibilities must be reassigned. The explicit distinction between roles addresses this question of division of responsibilities, thus improving the coordination and supporting tasks, and making the solution easily scalable regardless of the number of teachers. Moreover, this roles' model is particularly suitable in the case of social learning approaches.

The IMS-LD specification also promotes the structuring of activities. There are different types of activities in the PBL design: coordination activities (preparatory work, logistical tasks and evaluation steps, see Fig. 1), parallel activities (e.g., the students reading the CS description and the Facilitator giving support), and synchronization activities when the flow crosses roles (e.g., between the students discussing findings and the chair- person summarizing the findings). The use of IMS-LD specification also helps to distinguish between learning and support activities. Although this distinction is usually made, it is important that it should be made explicit in the pedagogical design from the very outset, to prevent the coordinator from becoming overloaded. This consideration is especially important in a PBL scenario, because the learners' interaction requires more attention.

The allocation of resources and environments is also made explicit when designing a pedagogical scenario using LD. In this PBL trial, several environments are defined to support group discussions (private and public forum, FAQ list, chat, e-mail, etc), both between the students, between students and the Facilitator, and between the Facilitator, Coordinator and Evaluator. It is worth noting that it is easier to think in terms of scalability and feasibility when the allocation is clearly defined from the outset. 


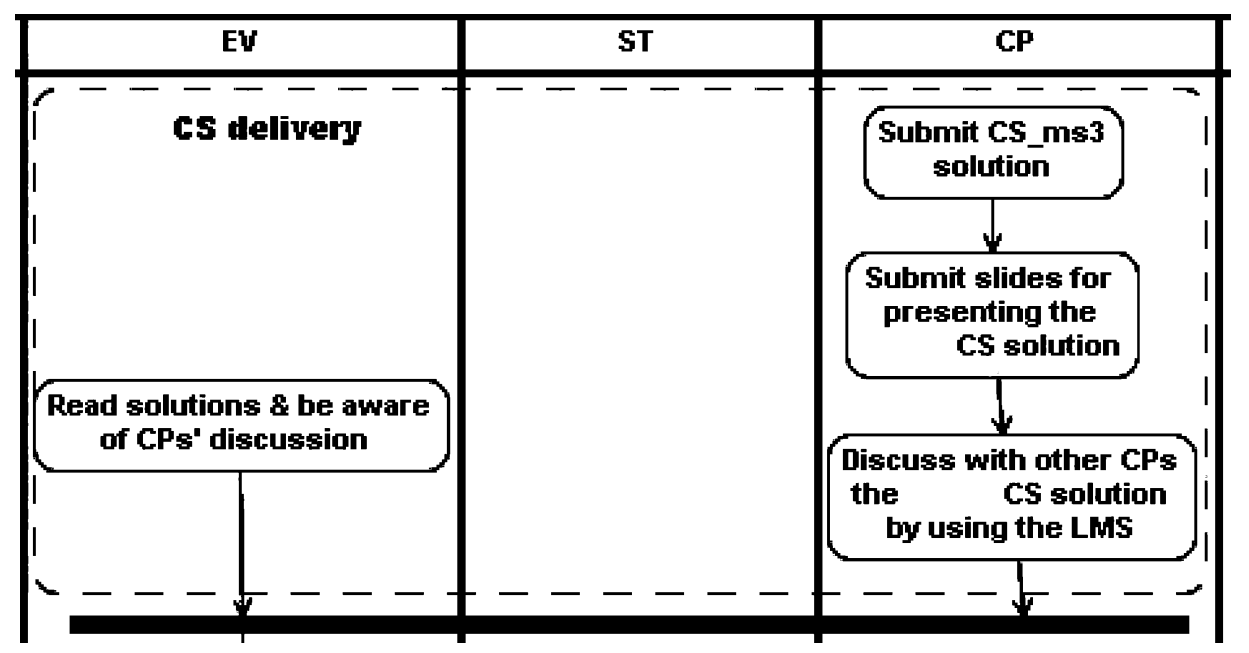

Fig. 6. Overall CS delivery.

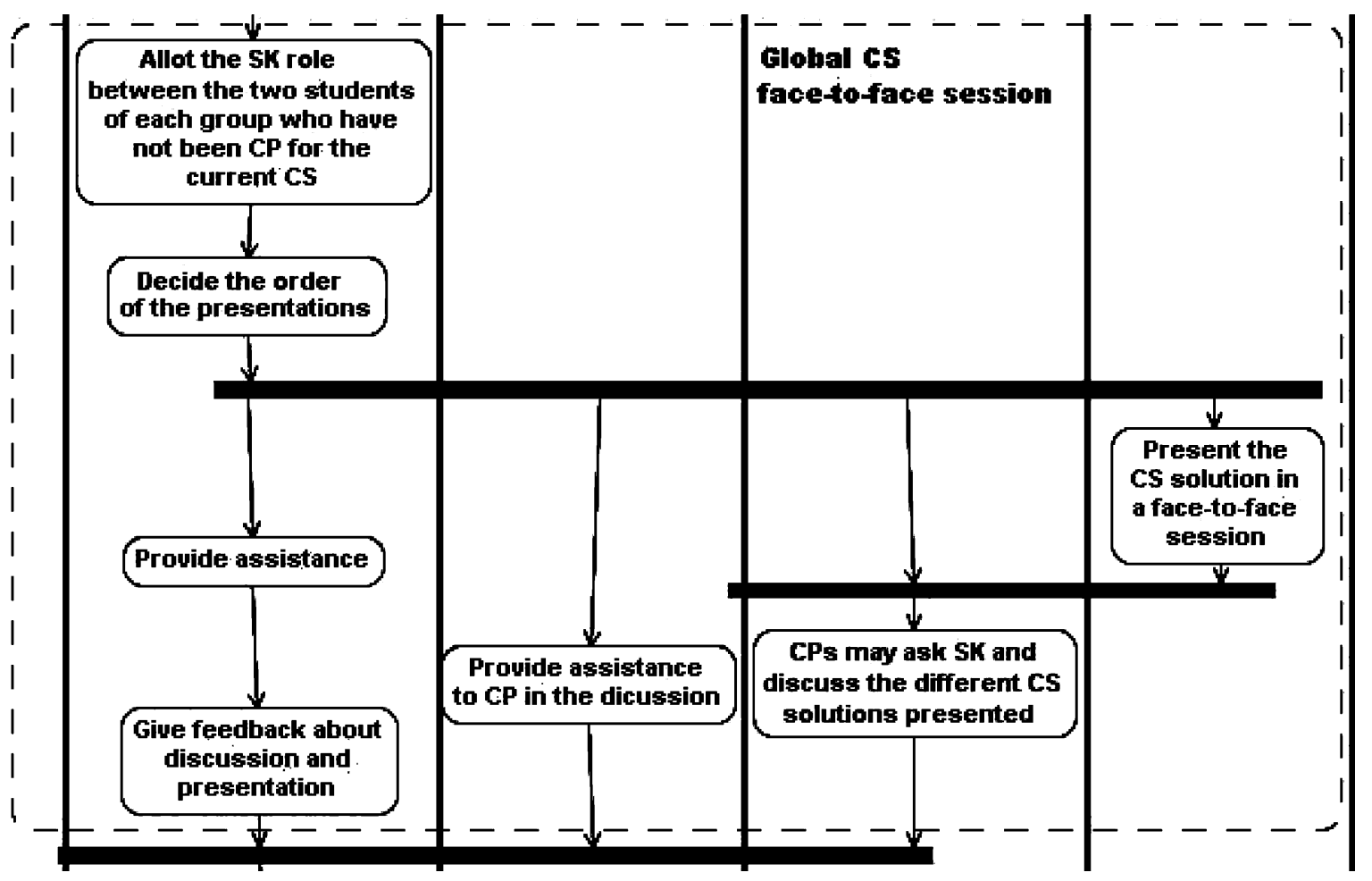

Fig. 7. Global CS face-to-face session

Reusability of the learning design is supported by the use of the IMS-LD standard notation and UML diagrams. In fact, the students who were involved in this PBL trial are so excited about the outcomes that most of them have asked teachers to use the same type of pedagogic approach for teaching the practical part of another course in the second term. Of course, teachers must review the design and implementation of the current PBL scenario and adapt it to the new course, but the design and adaptation costs are estimated to be very low, compared to the benefits of using LD.

Considering the long term, once LD methodology has been used for a substantial period of time, it should become possible to extract pedagogical patterns that help teachers identify the best teaching and learning practices, assisting them in reporting and performance analysis. Although just a prediction, this possibility clearly has great potential benefit that must be considered when adopting a cooperative strategy for innovation purposes in educational practices.

Some drawbacks have been observed with respect to the use of LD in this trial. The most important of these are as follows.

The initial effort needed to design a pedagogical trial using this methodology is likely to be greater than necessary when teachers think solely in terms of content, and split the responsibilities between the teachers involved.

A full implementation of the PBL trial using the corresponding IMS-LD XML binding [14] appears to be very costly for a blended learning framework. The IMS-LD specification was developed to support the design, implementation and 


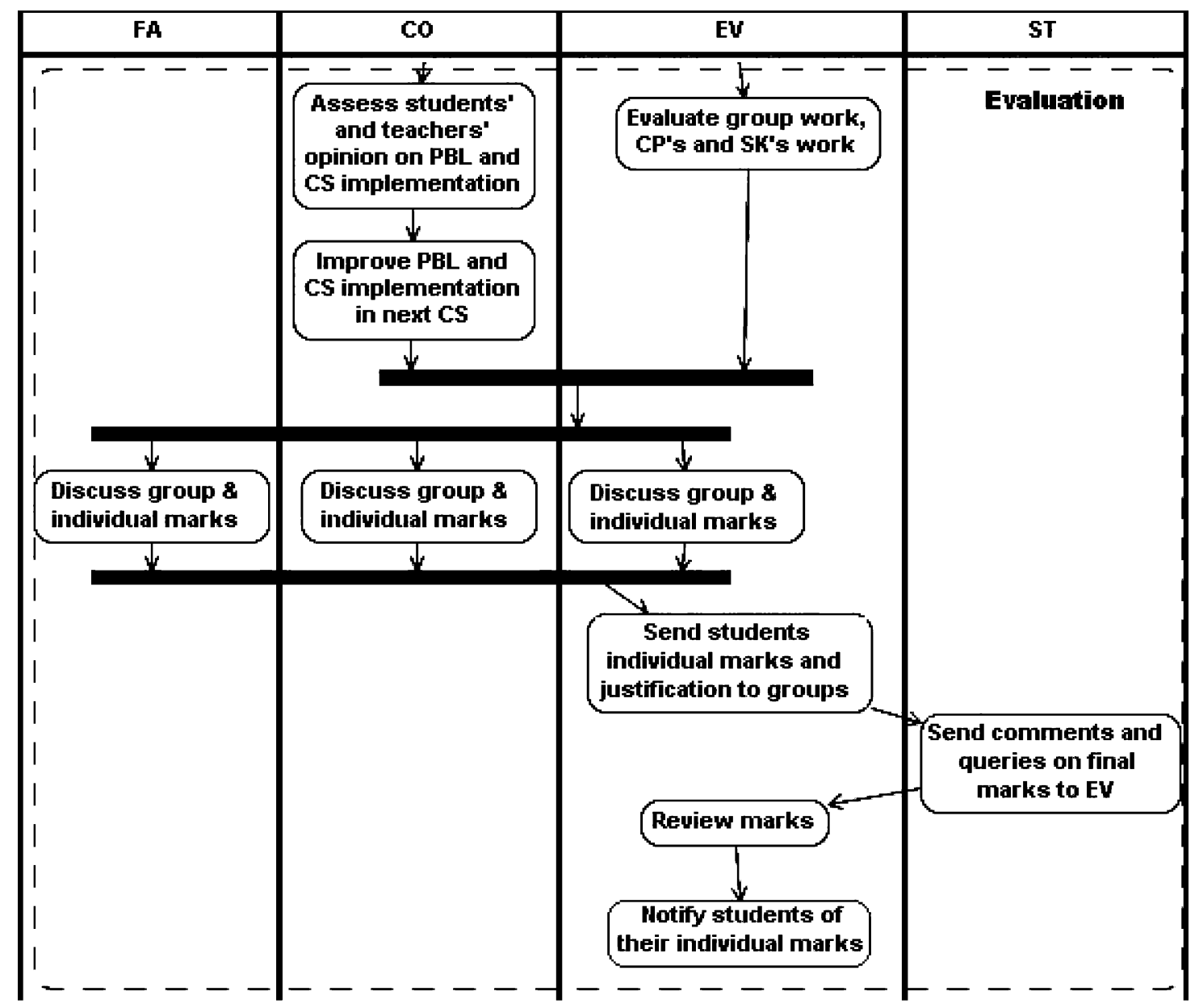

Fig. 8. Evaluation.

running of UoLs in eLearning environments. It would not be expected, therefore, that full adoption of the use of this specification would be easily addressed by teachers in blended learning. For one thing, a very high level of detail (related to the granularity of the specification) is required to make a machine-readable version of the UoL. Also, according to experts in this field that, editing tools should be separate from the specification, and specific to the teacher's needs [11], especially in a blended learning environment. But currently available tools for editing IMS-LD-compliant UoLs are still very demanding for most of those teachers. The teachers working in the department involved in this PBL trial are willing to be innovative in their teaching practices, as long as the increase in their workload is kept to a minimum. Since they have to combine teaching and research activities, the additional workload of implementing blended learning must be minimized. Bearing in mind these issues, the best solution may be to use IMS-LD just as an eLearning methodology.

The LD specification is divided into three parts, to make the task of implementation simpler. Level A provides the basic functionality required to define roles, resources and activities; Level B adds properties and conditions which support sophisticated adaptivity and interactivity; and Level $\mathrm{C}$ provides support for notification so that teachers, learners and other eLearning systems can be informed of progress in the learning activities
[12]. The implementation of a social learning model such as the PBL would involve the use of levels A, B, and C. Tackling the three levels of the specification in a blended learning environment (with limited human resources) still poses a challenge to teachers. Nevertheless, it is less difficult to keep the conceptual model in mind than it is to try and develop a machine-readable version of the corresponding PBL UoL.

The last two drawbacks can be easily solved by using the IMS-LD just as a methodology, and that was the decision taken before undertaking this trial.

\section{IMPLEMENTATION OF THE PBL}

The trial described in this paper is included in an undergraduate course. The subject Parallel Systems Architecture 1 (ASP1), which is divided into three parts: a) advanced memory hierarchy; b) pipeline processors design; and c) static/dynamic scheduling. To cover all the laboratory sessions of the syllabus with the PBL methodology, a CS was designed for each part, and divided into three milestones. The milestones for each CS were given to the students just after the theory explanations in the lectures. Students were inspired by having applied and finally understood concepts previously explained in lectures; this effect has also been pointed out in some recent studies [2].

The aim was that the PBL students should learn all the fundamental concepts of ASP1. Computer engineering content is 


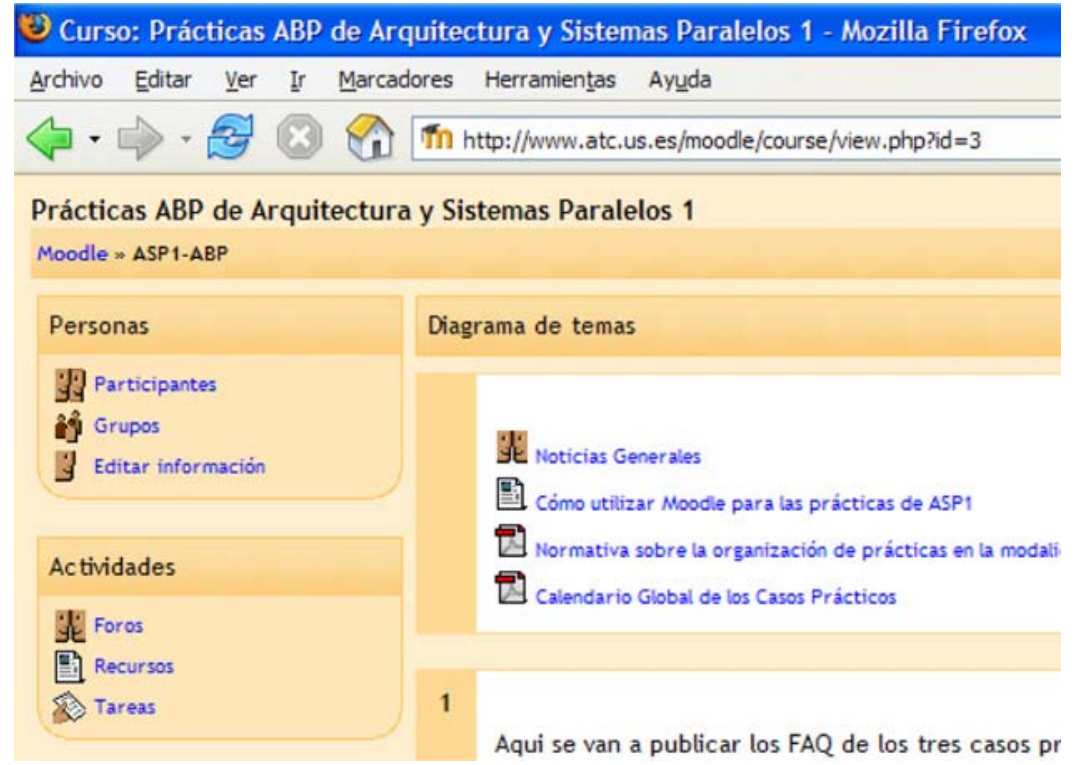

Fig. 9. LMS (Moodle) screenshot.

constantly changing, which means that graduating students must have the necessary knowledge to be able to design applications and use digital systems. Clearly, taking the goal of inculcating an in-depth knowledge of one single technology is not a correct teaching approach.

Another goal was to make the CS attractive to students. The success in reaching this goal is obvious, since the classical laboratory sessions used to be guided and intensive, and students were not able to propose creative solutions. Apart from the known advantages of PBL, the following additional aspects were incorporated: a) students felt they were supported efficiently by the facilitators when solving each PBL via Moodle LMS (see Fig. 9); b) the PBL laboratory classes were highly weighted because of the greater effort required (33\%, as opposed to $15 \%$ for the classical ones). According to the final results (see Section V), this objective was amply achieved.

The three CS were as follows:

- CS1: study of the influence of the miss rate of a cache memory and the execution time for several access patterns of the same data structure.

- CS2: analysis and study of the stall types for pipeline processors.

- CS3: Instruction Level Parallelism (ILP) extraction using static and dynamic scheduling.

As the objective of these CS was not to connect with other degree courses, but to apply a teaching strategy to transmit core concepts and, at the same time, promote the deployment of abilities and competences, any problems students may have had with the software tools (compiler flags, assembler language directives, simulators, etc.) needed to tackle each CS were always resolved by the Facilitators. In contrast, the objective concepts of each CS were not explained by the Facilitator, who just responded to specific queries, and advised students on the use of the complementary bibliography and lecture notes.

Another consequence of this distinction is that for some milestones the use of simulators was preferred. This approach pre- vented students from becoming bogged down with the complexity of using real systems. Furthermore, students could easily download their results from the simulators (directly or with little calculation). For example, it is well known that pipeline processor temporal diagrams are powerful tools for understanding pipeline working (hazards, stalls, ILP, and the like) [8], and the use of simulators is the only straightforward way to download these diagrams. However, due to the influence that the correct use of a tool has on the solution of any CS related to computer architecture, some questions related to the tools being used were always included as objectives of the weekly milestone.

Given the rigid scheduling of all syllabus courses, it was not feasible to maintain a weekly schedule. Because of students' other commitments such as exams, or work deadlines for other subjects, some milestones were delayed by one or two days, and once by a week. In general, deadline flexibility for milestones is something that needs to be taken into account in the design of a PBL for this kind of syllabus.

Apart from flexibility, the PBL development should have a very strict milestone scheduling. It is therefore necessary to have an electronic teaching tool available to allow rapid communication between participants: first, to clarify without delay any problems or questions that would stop a student from resolving a CS for a long period of time using the classical method (until the next lab session); second, to facilitate CS scheduling: initial regulations, weekly publication of the milestones, submission of the tasks, additional material requested by students (and so on); third, to extract other advantages from this kind of tool. In this trial, the following aspects proved important for facilitating CS solution and student evaluation:

- The creation of private forums (for each group) to discuss both technical aspects and problems related to the method. These forums were visited frequently by lecturers.

- The creation of a public FAQ for all the PBL students for each CS. Lecturers updated this FAQ with interesting questions from students. In this way the use of the tool is pro- 
moted and, at the same time, the level of skills and knowledge is enhanced in the groups.

- The log of student activity maintained by the software tool allows a fairer assessment to be made.

The overarching goal was to combine the advantages of lectures with those of e-learning tools. Moodle is an e-learning tool in which all the aforementioned tools can be easily integrated, and which furthermore offers a free license.

Lecturers participating in this kind of trial, where face-toface teaching and distance teaching are combined, and where e-learning tools play an active role, have to put in a lot of extra work on top of their normal workload. Specifically, they have to learn a new method, continuously changing their role (some of them are not present at the University of Seville as facilitators); they have to prepare two different laboratory sessions (for PBL students and classical ones); they have to answer a steady stream of forum questions about each CS; and on top of this they have to meet with many more students in their offices.

As there were three CS, groups of three students were chosen. Therefore, each was able to play a different role in each CS, as well as collaborating together in the resolution of the CS. Normally, when students are grouped together to solve several problems, they split the work up, so that only one of them would have undertaken all the work of each CS. With this in mind, the authors developed a strategy to stop collaboration becoming a matter of task division between learners [17]. Overall, rotation control of students' roles was rigid.

Students were therefore expected to play different roles to develop a wider set of abilities, but without overloading any student with the resolution of the complete CS covering that whole area of the course. In the first CS the students drew lots for the coordinator role. However, the speaker role was allotted at the end of the CS, to stimulate all the students equally.

The face-to-face sessions $(1 \mathrm{~h} / \mathrm{wk})$ were useful for controlling the development of each group and contrasting opinions. These sessions allowed students to correct or criticize the proposals from other groups. This activity was taken into account by the lecturers in calculating the final mark. Finally, the last face-to-face session was the oral presentation of the whole CS by the Speaker of each group and the subsequent debate about the solutions proposed and the mistakes made. The lecturers had the task of correcting the mistakes found in the oral presentation, both conceptually and in oratory, in terms of underlying concepts and of presentation. This approach encouraged speakers to discover their own mistakes, to improvise in their presentation and to change their presentation spontaneously depending on the facilitator's suggestions. Finally, the lecturer invited the participation of the coordinators, in order to assess their intervention.

\section{Evaluation of the PBL}

An important aspect of this trial was the evaluation of the method by the students. This evaluation has provided useful data, which will help to improve the method for future courses, to validate the adopted blended learning and to test the correctness of the PBL design based on e-learning standards.

The first relevant fact concerned students' choice of the PBL option (see Section III). The course (ASP 1) selected for this trial has roughly 300 students enrolled, not all of whom are full-time students (many of them have jobs). The actual number of students who attend both theoretical and lab classes is around 180 . Only 15\% (28 students) of the total enrolment opted for the PBL method, while most of them rejected it. It is reasonable to conclude that the majority of students prefer not to try new learning methodologies, maybe because they are unfamiliar with them.

Two questionnaires were given to the PBL students. Additionally, the personal opinions of a significant sample of students (both PBL and non-PBL) were gathered. Their corresponding responses were very positive. The main result was that a significant number of the next enrolment's students asked for to enroll in the PBL trial, even before the details of this method were presented to them. They had heard about this method from the previous year's students, who described PBL's benefits and the stimulus that this methodology produced on their understanding of the course. The high level of satisfaction on the part of the students who learnt with the PBL scenario, proved that they consider this method to be a viable way to improve their competencies and abilities.

Due to the success of the PBL method in that pilot experience, it was decided to apply the same pedagogic design approach in another course called "Programming Techniques for Advanced Architectures," which is part of a specialization Master taught at the same Higher Technical School of Computer Engineering. More useful feedback was obtained at the end of that second pilot experience. The application of the pedagogic method was so much appreciated by the students that at the end of the teaching period they marked it as the most interesting of all the Master's-level courses.

The completion of the two questionnaires by PBL students was voluntary and anonymous. The final questionnaire was presented at the end of the trial, but before the final theoretical exam. Both the design of the questionnaire, and the collection and processing of the results, were performed by a lecturer not involved in teaching the course, to maintain the objectivity of the survey.

The questionnaire was divided into three parts: a) questions about the teaching environment; b) questions about the methodology; and c) questions about the development of the case study.

$82 \%$ of the students completed the questionnaire and the results were encouraging for the lecturers. For the first group of questions, the students valued the usefulness of the LMS tool at 3.7 and 4.04 (from 1 to 5), respectively. It is clear that students consider that Moodle is an easy to use software tool, and very useful as LMS.

The second group was centered on the methodology. Fig. 10 shows the results of their opinion as to which skills they think they have practiced most and which they have valued most.

The results of this second group of responses indicate that students think that this trial is focused mainly on oral presentation, and considers collaboration as being unimportant, which demonstrates students' lack of awareness of PBL methodology and the association of evaluation with knowledge instead of competences or skills.

Furthermore, they had to indicate how much they considered that the PBL methodology had contributed to their learning, in contrast to the classical method, compared to other degree 


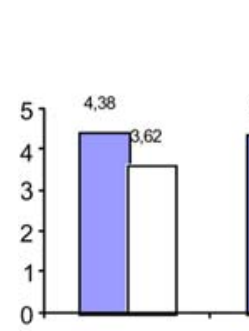

(a)
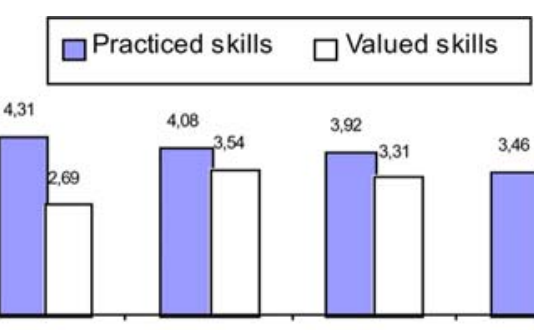

(b)

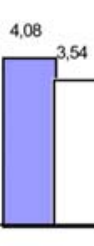

(c)

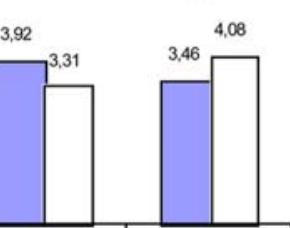

(d)

(e)
Fig. 10. Skills assessment. (a) Acquisition of knowledge. (b) Collaboration between classmates. (c) Decision-making. (d) Discussion. (e) Oral presentations. (1-Hardly practiced/very little valued, 5-Continuously practiced/highly valued). In all the results, the variance is less than 1.

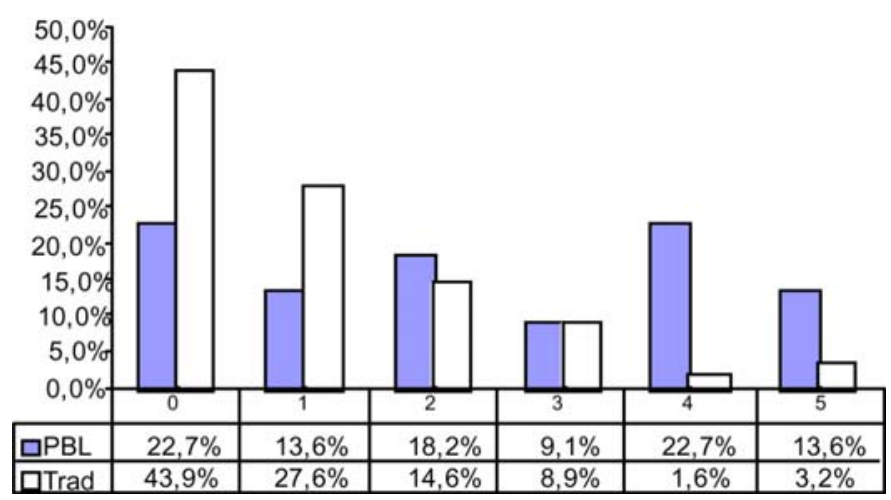

Fig. 11. Final exam marks for each methodology. Of the 28 PBL students, 22 took the exam. Of the 152 classic methodology students, 123 took the exam. 0 $=$ "fail," $1=$ "pass" to $5=$ "excellent."

courses (1-Less significant, 5-Much more significant). They answered with 4.69 on average (variance $=0.4$ ).

The third group of questions was related to the development of the practical case and how this could influence student learning. Asked whether they thought they had a better chance of passing the exam because of having met all the practical CS milestones, 92\% answered "yes." As for their opinion (from 1 to 5) about the overall influence of the PBL trial for their learning, they evaluated it at 4.07 on average (variance $=1,3$ ).

Finally, the seven students who were repeating their second year were asked to compare the classical laboratory sessions of this course with those of this new pedagogic model. They were asked if they believed that PBL methodology contributed to a better understanding of the subject than the classical one. They all answered "yes."

The last important consideration is the marks comparison (PBL versus classical students), as the written final exam was the same for both. The results are shown in Fig. 11.

The marks for the PBL students are considerably better than those of their classical counterparts. While PBL students' believe this method has helped them, it is possible that the difference in marks may also be due to the PBL selection method. The students who chose PBL may have been more motivated than the others, or they may have worked harder in this course.

\section{CONCLUSION}

Students have accepted the PBL model in a natural way, evaluating the trial with very positive feedback. It is noteworthy that they believed that the new methodology would help them to learn and get through the course better than by using the traditional approach.

Thanks to the use of IMS-LD, the PBL scenario design was made explicit, and is therefore easily reusable for the same or even other courses. In fact, the authors are currently repeating the trial in other related courses.

Additional benefits of using the standard mean that scalability and feasibility concerns can be analyzed in advance, thereby improving the division of responsibilities, activities structuring and real practice support. In the future, the authors expect to be able to use their own pool of learning designs to extract pedagogical patterns that help them to identify the best teaching and learning practices. The authors believe that the global adoption of IMS-LD methodology should be considered as a strategic decision in the long term.

\section{ACKNOWLEDGMENT}

The authors would like to thank R. Koper and his team (in particular, C. Tattersall and D. Burgos) for making it possible for one of the authors to stay at the OUNL from March-April 2006 and making a feasibility survey on the use of IMS-LD to support PBL in a blended learning framework, prior to this trial.

\section{REFERENCES}

[1] J. H. C. Moust et al., "Signs of erosion: Reflections on three decades of problem-based learning at Maastricht University," Higher Educ., vol. 50, pp. 665-683, Nov. 2005.

[2] H. J. M. Dolmans et al., "Problem-based learning: future challenges for educational practice and research," Med. Educ., vol. 39, no. 7, pp. 732-741, Jul. 2005.

[3] M. A. Albanese and S. S. Mitchell, "Problem-based learning: A review of literature on its outcomes and implementation issues," Acad. Med., vol. 68, no. 1, pp. 52-81, Jan. 1993.

[4] F. Dochy et al., "Effects of problem-based learning: a meta-analysis," Learn. Instruc., vol. 13, no. 5, pp. 533-568, Oct. 2003.

[5] "Design-based research: an emerging paradigm for educational inquiry," Educ. Res., vol. 32, no. 1, pp. 5-8, Jan. 2003.

[6] IMS Global Learning Consortium, Learning Design Specification, 2008 [Online]. Available: http:www.imsproject.org/learningdesign/index.html

[7] R. Koper, "Current research in learning design," J. Educ. Technol. Soc., vol. 9, no. 1, pp. 13-22, Jan. 2006.

[8] Moodle Trust, 2008 [Online]. Available: http://moodle.org

[9] European Commission, The Declaration of Bologna, 2007 [Online]. Available: http://ec.europa.eu/education/policies/educ/bologna/ bologna_en.html

[10] R. Koper and B. Olivier, "Representing the learning design of units of learning," J. Educ. Technol. Soc., vol. 7, no. 3, pp. 97-111, 2004.

[11] D. Griffiths et al., "Learning design tools," in Learning Design: A Handbook on Modelling and Delivering Networked Education and Training, R. Koper and C. Tattersall, Eds. Berlin, Germany: Springer-Verlag, 2005, pp. 109-135.

[12] The UNFOLD Project: Understanding and Using Learning Design, D. Burgos and D. Griffiths, Eds., 1st ed. Heerlen, Netherlands: Open Univ. Netherlands, 2005.

[13] IMS Best Practice and Implementation Guide, IMS Global Learning Consortium, Inc., 2003 [Online]. Available: http://www.imsglobal.org/ learningdesign/ldv1p0/imsld_bestv1p0.html

[14] IMS LD XML Binding, IMS Global Learning Consortium, Inc., 2003 [Online]. Available: http://www.imsglobal.org/learningdesign/ldv1p0/ imsld_bindv1p0.html

[15] J. L. Hennessy and D. A. Patterson, Computer Architecture. A Quantitative Approach, 4th ed. San Francisco, CA: Morgan-Kaufmann, 2006.

[16] M. Savin-Baden, Problem-Based Learning in Higher Education: Untold stories. Buckingham, U.K.: Open Univ. Press/SRHE, 2000. 
[17] P. Dillenbourg et al., "The evolution of research on collaborative learning," in Learning in Humans and Machines: Towards an Interdisciplinary Learning Science, E. Spada and P. Reiman, Eds. Oxford, U.K.: Elsevier, 1996, pp. 189-211. 